\title{
A GENERALIZED INTERFACE LAW IN DYNAMIC COUPLED THERMOELASTICITY: ASYMPTOTIC ANALYSIS AND FEM VALIDATION
}

\author{
M. Serpilli ${ }^{1 *}$, S. Dumont ${ }^{2}$, R. Rizzoni ${ }^{3}$ and F. Lebon ${ }^{4}$ \\ ${ }^{1}$ Department of Civil and Building Engineering, and Architecture, Universitá Politecnica delle \\ Marche, Ancona, Italy \\ 2 IMAG CNRS UMR 5149, University of Nîmes, Nîmes, France \\ Department of Engineering, University of Ferrara, Ferrara, Italy \\ Laboratoire de Mècanique et d?Acoustique, CNRS, Aix-Marseille University, Marseille, France \\ *m.serpilli@univpm.it
}

The present work deals with the modeling of interfaces in coupled thermoelasticity by means of the asymptotic analysis (see, e.g., $[1,2,3]$ ). The composite body is constituted by two thermoelastic media (adherents) bonded together by a thin intermediate thermoelastic layer (adhesive). By considering a small parameter $\varepsilon$, associated with the thickness of the adhesive, we apply the asymptotic expansions method to the thermoelastic evolution problem by letting $\varepsilon$ tends to zero. We characterize different limit interface laws, considering also the contribution of the higher order terms of the expansion. The cases of soft (lowly-conduting) and hard (moderately conducting) interfaces are considered: the first is derived assuming the constitutive coefficients linearly depending on the thickness $\varepsilon$; the second considers the material properties independent of $\varepsilon$. By using the approach developed in [4] for multiphysic materials, we also identify a general thermoelastic interface law which comprises the above aforementioned models. Numerical investigations are performed in the framework of the finite element method, by comparing the results obtained by modeling the adhesive as a continuum material (discretized in finite elements even in the thickness) with the results carried out using the derived interface models.

\section{References}

[1] P.G. Ciarlet (1997), Mathematical Elasticity, Vol. II: Theory of Plates. North-Holland, Amsterdam.

[2] M. Serpilli, (2017). Asymptotic interface models in magneto-electro-thermo-elastic composites. Meccanica, 52 (6), 1407-1424.

[3] S. Dumont, R. Rizzoni, F. Lebon andE. Sacco, (2018). Soft and hard interface models for bonded elements, Composite Part B, 153, 480-490.

[4] M.Serpilli R. Rizzoni, F. Lebon and S. Dumont,(2019). An asymptotic derivation of a general imperfect interface law for linear multiphysics composites, Int. J. Solids Struct. 180-181, 97-107. 